\title{
MARTINGALES IN THE LIMIT AND AMARTS
}

\author{
G. A. EDGAR AND L. SUCHESTON ${ }^{1}$
}

\begin{abstract}
The notion of amart is compared to that of a martingale in the limit and game fairer with time. Every real-valued amart is a martingale in the limit. More generally, a Banach space $E$ is finite-dimensional if and only if every $E$-valued amart is a martingale in the limit (or a game fairer with time). Several crucial properties possessed by amarts fail both for martingales in the limit and games fairer with time: the maximal inequality, the optional stopping theorem, the optional sampling theorem, the Riesz decomposition; therefore a general theory analogous to the amart theory cannot be based on the notion of a martingale in the limit. It is also observed that either the optional sampling theorem or a weak form of the Riesz decomposition must fail for any class of sequences of random variables strictly larger than the class of amarts.
\end{abstract}

1. Let $(\Omega, \mathscr{F}, P)$ be a complete probability space, $\left(\mathscr{F}_{n}\right)$ an increasing sequence of $\sigma$-algebras. A stopping time is a random variable $\tau$ assuming positive integer values and the value $+\infty$, and such that for each $n$, $\{\tau=n\} \in \mathscr{F}_{n}$. The collection of all bounded stopping times is denoted by $T$. An amart is a sequence of integrable random variables $X_{n}$, adapted to the $\mathscr{F}_{n}$ 's and such that $\left(E X_{\tau}\right)_{\tau \in T}$ converges. A martingale in the limit is an adapted sequence of integrable random variables such that

$$
\lim _{n \rightarrow \infty} \sup _{m>n}\left|E\left[X_{m} \mid \mathscr{F}_{n}\right]-X_{n}\right|=0 \text { a.s. }
$$

In the sequel the words 'a.s.' may be omitted, and relations and formulas will be understood to hold modulo sets of probability zero.

Both amarts and martingales in the limit generalize martingales. An amart theory parallel to Doob's martingale theory was developed in [8]. Recently A. G. Mucci, who introduced martingales in the limit [13], has proved that $L_{1}$-bounded martingales in the limit converge a.s. [14]. This is an interesting result, because martingales in the limit constitute a considerable generalization of martingales. In particular, it will be shown below that every amart is a martingale in the limit. This naturally raises the question whether a general theory can be built around the notion of the martingale in the limit. We believe that the present paper provides a negative answer to this question. We show that several crucial properties possessed by amarts fail for

Received by the editors April 6, 1977.

AMS (MOS) subject classifications (1970). Primary 60G40, 60G45, 60G99.

Key words and phrases. Martingale in the limit, amart, maximal inequality, optional sampling theorem, game fairer with time.

'The research of this author is in part supported by the National Science Foundation. 
martingales in the limit: the maximal inequality, Riesz decomposition, optional stopping theorem, optional sampling theorem. These properties are also in default for another, weaker, notion: that of a game fairer with time, introduced by L. H. Blake [3].

We essentially restrict our discussion to the real case, but we show at the end of the paper that amarts taking values in a Hilbert space, unlike the real ones, need not be martingales in the limit. In fact, a Banach space $E$ is finite dimensional if and only if every $E$-valued amart is a martingale in the limit (or a game fairer with time).

2. THEOREM 1. Every real-valued amart is a martingale in the limit.

Proof. Suppose that $\left(X_{n}, \mathscr{F}_{n}\right)$ is not a martingale in the limit. Then there exist $\alpha, \delta>0$ such that

$$
P\left\{\limsup _{n \rightarrow \infty}\left(\sup _{m>n}\left|E\left[X_{m} \mid \mathscr{F}_{n}\right]-X_{n}\right|\right)>\delta\right\}>2 \alpha .
$$

Therefore, either

$$
P\left\{\limsup _{n \rightarrow \infty}\left(\sup _{m>n}\left(E\left[X_{m} \mid \mathscr{F}_{n}\right]-X_{n}\right)\right)>\delta\right\}>\alpha
$$

or

$$
P\left\{\limsup _{n \rightarrow \infty}\left(\sup _{m>n}\left(X_{n}-E\left[X_{m} \mid \mathscr{F}_{n}\right]\right)\right)>\delta\right\}>\alpha .
$$

Since $\left(X_{n}\right)$ may be replaced by $\left(-X_{n}\right)$, we may assume that (1) holds. Let a positive integer $N$ be given. Then

$$
P\left\{(\exists n \geqslant N)(\exists m \geqslant n) E\left[X_{m} \mid \mathscr{F}_{n}\right]-X_{n}>\delta\right\}>\alpha,
$$

so there exists $N^{\prime}, N<N^{\prime}$, such that

$$
P\left\{\exists n, m ; N \leqslant n \leqslant m \leqslant N^{\prime} ; E\left[X_{m} \mid \mathscr{F}_{n}\right]-X_{n}>\delta\right\}>\alpha .
$$

If $N<n<N^{\prime}$, define

$$
\begin{gathered}
A_{n}=\left\{\exists m, n \leqslant m \leqslant N^{\prime}, E\left[X_{m} \mid \mathscr{F}_{n}\right]-X_{n}>\delta\right\}, \\
A=\bigcup_{n=N}^{N^{\prime}} A_{n} .
\end{gathered}
$$

Thus $A_{n} \in \mathscr{F}_{n}$ for all $n$, and $P(A)>\alpha$. Define $\tau_{1}, \tau_{2}$ by

$$
\begin{gathered}
\tau_{1}(\omega)= \begin{cases}\min \left\{n: N \leqslant n \leqslant N^{\prime}, \omega \in A_{n}\right\} & \text { for } \omega \in A, \\
N^{\prime} & \text { for } \omega \notin A ;\end{cases} \\
\tau_{2}(\omega)=\left\{\begin{array}{c}
\min \left\{m: n \leqslant m \leqslant N^{\prime}, E\left[X_{m} \mid \mathscr{F}_{n}\right]-X_{n}>\delta\right\} \\
\quad \text { for } \omega \in A_{n} \backslash\left(A_{N} \cup A_{N+1} \cup \cdots \cup A_{n-1}\right), \\
N^{\prime} \text { for } \omega \notin A .
\end{array}\right.
\end{gathered}
$$

Now given $n, m$, we have $\left\{\tau_{1}=n, \tau_{2}=m\right\} \in \mathscr{F}_{n}$. Thus, 


$$
\begin{aligned}
E\left[X_{\tau_{2}}\right]-E\left[X_{\tau_{1}}\right] & =\sum_{n=N}^{N^{\prime}} \sum_{m=n}^{N^{\prime}} E\left[\left(X_{m}-X_{n}\right) 1_{\left\{\tau_{1}=n, \tau_{2}=m\right\}}\right] \\
& =\sum_{n=N}^{N^{\prime}} \sum_{m=n}^{N^{\prime}} E\left[\left(E\left[X_{m} \mid \mathscr{F}_{n}\right]-X_{n}\right) 1_{\left\{\tau_{1}=n, \tau_{2}=m\right\}}\right] \\
& =E\left[\left(E\left[X_{m} \mid \mathscr{F}_{n}\right]-X_{n}\right) 1_{A}\right] \geqslant \delta \alpha .
\end{aligned}
$$

Hence, for every positive integer $N$, there exist $\tau_{1}, \tau_{2} \in T, N \leqslant \tau_{1} \leqslant \tau_{2}$, such that $E\left[X_{\tau_{2}}\right]-E\left[X_{\tau_{1}}\right] \geqslant \delta \alpha$, so $\left(X_{n}\right)$ is not an amart.

3. The defining property of martingales in the limit can be restated as follows. The double sequence

$$
H_{n, m}=E\left[\left.X_{m}\right|^{F_{n}}\right]-X_{n}
$$

converges to zero a.s. when $m \geqslant n \rightarrow \infty$. An adapted sequence $\left(X_{n}, \mathscr{F}_{n}\right)$ is called a game fairer with time iff $H_{n, m}$ converges to zero in probability as $m>n \rightarrow \infty$. Clearly every martingale in the limit is a game fairer with time. It was observed in [9] that amarts are games fairer with time, but not vice versa.

THEOREM 2. (i) The maximal inequality [7], [8] fails for martingales in the limit. More precisely, there exists an $L^{1}$-bounded martingale in the limit $\left(X_{n}\right)$ such that $\sup _{\lambda>0} \lambda P\left(\sup \left|X_{n}\right|>\lambda\right)=\infty$.

(ii) The Riesz decomposition (in the weakest form assuring uniqueness) fails for martingales in the limit. That is, there exists a martingale in the limit $\left(X_{n}, \mathscr{F}_{n}\right)$ which cannot be represented in the form $X_{n}=Y_{n}+Z_{n}$, where $\left(Y_{n}, \mathscr{F}_{n}\right)$ is a martingale, $\int_{A} Z_{n} \rightarrow 0 \forall A \in \cup_{n} \mathscr{F}_{n}$.

(iii) The optional stopping theorem fails both for martingales in the limit and games fairer with time. More precisely, there exists a martingale in the limit $\left(X_{n}, \mathscr{F}_{n}\right)$, and a stopping time $\sigma$ with $P(\sigma=\infty)>0$, such that $\left(X_{n \wedge \sigma}, \mathscr{F}_{n \wedge \sigma}\right)$ is not a game fairer with time (and a fortiori not a martingale in the limit).

(iv) The optional sampling theorem (in the weakest form) fails both for martingales in the limit and for games fairer with time. More precisely, there exists a martingale in the limit $\left(X_{n}, \mathscr{F}_{n}\right)$ and a sequence of bounded stopping times $\tau_{n}$ with $\tau_{n} \uparrow \infty$ uniformly, such that $\left(X_{\tau_{n}}, \mathcal{F}_{\tau_{n}}\right)$ is not a game fairer with time.

Proof. (i) Let $\left(A_{i}\right)$ be a measurable partition of $\Omega$ with $P\left(A_{i}\right)=1 / i-1 /(i$ $+1), i=1,2, \ldots$ Let $X_{i}=i(i+1) 1_{A_{i}}$, and $\mathscr{F}_{i}=\sigma\left(X_{1}, \ldots, X_{i}\right)(=$ the $\sigma$-algebra generated by $X_{1}, \ldots, X_{i}$ ). Then $E X_{i}=1$, but

$$
i(i+1) P\left(\sup _{n}\left|X_{n}\right| \geqslant i(i+1)\right)=i(i+1) \sum_{n>i} P\left(A_{n}\right)=i+1 \rightarrow \infty .
$$

$\left(X_{n}, \widetilde{F}_{n}\right)$ is a martingale in the limit, because on the set $\cup_{i<n} A_{i}$,

$$
E\left[X_{m} \mid \mathscr{F}_{n}\right]-X_{n}=0 \text { a.s., and } \lim _{n}\left(\bigcup_{i<n} A_{i}\right)=\Omega \text {. }
$$

(ii) Let $X_{n}$ converge a.s., $\mathscr{F}_{n}=\sigma\left(X_{1}, X_{2}, \ldots\right)=\mathscr{F}_{1}$, and assume that there 
is a set $A \in \cup \mathscr{F}_{m}$ such that $\int_{A} X_{n}$ does not converge. Then $\left(X_{n}, \mathscr{F}_{n}\right)$ is a martingale in the limit which has no Riesz decomposition.

(iii) Let $\left(A_{n}\right)$ be independent events with $P\left(A_{1}\right)=0, P\left(A_{n}\right)=1 / n^{2}$ for $n>1$. Define $X_{n}=n 1_{A_{n}}, \mathscr{F}_{n}=\sigma\left(X_{1}, \ldots, X_{n}\right)$. By the Borel-Cantelli lemma, $X_{n} \rightarrow 0$ a.s. If $n<m, E^{\mathfrak{S}_{n}} X_{m}-X_{n}=E X_{m}-X_{n} \rightarrow 0$ a.s., hence $\left(X_{n}, \mathscr{F}_{n}\right)$ is a martingale in the limit. Let $\sigma=\inf \left\{n: X_{n} \neq 0\right\}$; then $P(\sigma=\infty)=\prod_{n=2}^{\infty}(1-$ $\left.n^{-2}\right)=1 / 2$. Let $Y_{n}=X_{\sigma \wedge n}, \mathcal{G}_{n}=\mathscr{F}_{\sigma \wedge n}$. Let $\omega \in\{\sigma=\infty\}$; we claim that for each $n$,

$$
\sup _{m>n}\left|E\left[Y_{m} \mid \mathcal{G}_{n}\right]-Y_{n}\right|(\omega)=+\infty
$$

hence $\left(X_{n}, \mathscr{F}_{n}\right)$ is not a game fairer with time. Let $M$ be a positive constant, and choose $m>n$ such that $\frac{1}{2} \sum_{k=n+1}^{m} k^{-1} \geqslant M$. Now $\omega \in A_{2}^{c} \cap \cdots \cap A_{n}^{c}$, an atom of $\mathcal{G}_{n}$, and $E\left[Y_{m} \mid \mathcal{G}_{n}\right](\omega)$ is the average value of $Y_{m}$ on this atom. Then

$$
E\left[Y_{m} \mid \mathcal{S}_{n}\right](\omega)=\sum_{k=n+1}^{m} k\left[\prod_{j=n+1}^{k-1}\left(1-\frac{1}{j^{2}}\right)\right] \frac{1}{k^{2}} \geqslant \frac{1}{2} \sum_{k=n+1}^{m} \frac{1}{k} \geqslant M .
$$

Since $M$ is arbitrary and $Y_{n}(\omega)=0$, (3) follows.

(iv) Let $\left(X_{n}, \mathscr{F}_{n}\right)$ be as in (iii). Set $N_{1}=1$. Given $N_{k}$ and a positive constant $M$, let $N_{k+1}>N_{k}$ be the smallest integer such that $\frac{1}{2} \sum_{n=-N_{k}+1}^{N_{k+1}} n^{-1} \geqslant M$. Define $\tau_{k} \in T$ by

$$
\tau_{k}=\left\{\begin{array}{c}
\inf \left\{n: N_{k}+1 \leqslant n \leqslant N_{k+1}, X_{n} \neq 0\right\} \\
\quad \text { if this set is not empty, } \\
N_{k+1} \text { otherwise. }
\end{array}\right.
$$

Let $Y_{k}=X_{\tau_{k}}, \mathcal{G}_{k}=\mathscr{F}_{\tau_{k}}$; then the $\sigma$-algebras $\mathcal{G}_{k}$ are independent, hence

$$
\begin{aligned}
E\left[Y_{k} \mid \mathcal{G}_{k-1}\right] & =E\left[Y_{k}\right]=\sum_{n=N_{k}+1}^{N_{k+1}} n P\left(Y_{k}=n\right) \\
& =\sum_{n=N_{k}+1}^{N_{k+1}} n\left[\prod_{m=N_{k}+1}^{n-1}\left(1-\frac{1}{m^{2}}\right)\right] \frac{1}{n^{2}} \geqslant \frac{1}{2} \sum_{n=N_{k}+1}^{N_{k+1}} \frac{1}{n} \geqslant M .
\end{aligned}
$$

Since $M$ is arbitrary and $Y_{n} \rightarrow 0$ a.s.,

$$
\lim _{m>n \rightarrow \infty} E\left[Y_{m} \mid \mathscr{F}_{n}\right]-Y_{n}=+\infty \text { a.s. }
$$

Hence $\left(Y_{n}, \mathscr{F}_{n}\right)$ is not a game fairer with time.

4. Remarks. (a) The example in the proof of Theorem 2(iv) can be generalized as follows. Let $X_{n}$ be independent integrable real random variables such that $X_{n} \rightarrow 0$ a.s. and $E X_{n} \rightarrow 0$. Let $\mathscr{F}_{n}=\sigma\left(X_{1}, \ldots, X_{n}\right) ;\left(X_{n}, \mathscr{F}_{n}\right)$ is a martingale in the limit. Now suppose $E \sup \left|X_{n}\right|=+\infty$; this happens in (iv) above, and also in the following example: $X_{n}=Y_{n} / n$, where $Y_{n}$ are positive, independent, identically distributed random variables, with $E Y_{1}<$ $\infty, E\left(Y_{1} \log ^{+} Y_{1}\right)=\infty$ (cf. Burkholder [5], and McCabe and Shepp [12]). By [11, Theorem 3.3], $\left(E X_{\tau}\right)_{\tau \in T}$ is not bounded, hence there exists $\tau_{1} \in T$ such 
that $\left|E X_{\tau_{1}}\right| \geqslant 2$. Apply the same theorem to the sequence $\left(X_{n}\right)_{n>\sup \tau_{1}}$ to obtain a stopping time $\tau_{2}>\tau_{1}$ with $\left|E X_{\tau_{2}}\right|>2^{2}$. Then apply the same theorem to the sequence $\left(X_{n}\right)_{n>\sup \tau_{2}}$, etc. Then $\tau_{n} \uparrow \infty$ (uniformly), $\left|E X_{\tau_{n}}\right| \rightarrow$ $\infty$, and the $\sigma$-algebras $\mathscr{F}_{\tau_{n}}$ are mutually independent. Hence

$$
\lim _{n>m \rightarrow \infty}\left|E\left[X_{\tau_{m}} \mid \mathscr{F}_{\tau_{n}}\right]\right|=\lim _{n>m \rightarrow \infty}\left|E X_{\tau_{m}}\right|=+\infty
$$

Since $X_{\tau_{n}} \rightarrow 0$ a.s., it follows that $\left(X_{\tau_{n}}, \mathscr{F}_{\tau_{n}}\right)$ is not a game fairer with time.

(b) If $\sup _{n}\left|X_{n}\right| \in L^{1},\left(X_{n}, \mathscr{F}_{n}\right)$ is a martingale in the limit if and only if $X_{n}$ converges a.s., which is true if and only if $\left(X_{n}, \mathscr{F}_{n}\right)$ is an amart. The four properties considered in Theorem 2 are then easily seen to hold. For the case of a game fairer with time under the assumption $\sup _{n}\left|X_{n}\right| \in L^{1}$, see Blake [4].

(c) A Banach-valued amart need not be a martingale in the limit. In fact, more is true; the following statement is modelled on Alexandra Bellow's remarkable characterization of Banach spaces in which amarts converge strongly as finite dimensional [2]. If $E$ is a Banach space such that every $E$-valued amart is a martingale in the limit, then $E$ is finite dimensional.

SKETCH OF PROOF. Let at first $E=l_{2}$. A variant of the amart given in [7] and [8], which converges weakly but not strongly, is not a martingale in the limit: Let $\left\{e_{n}^{i}, n=1,2, \ldots ; 1 \leqslant i \leqslant 2^{n}\right\}$ be the standard orthonormal basis for $l_{2}$, set $X_{n}=\sum_{i=1}^{2^{n}} e_{n}^{i} 1_{A_{n}^{i}}$, where $A_{n}^{i} \cap A_{n}^{j}=\varnothing$ if $i \neq j$, and $P\left(A_{n}^{i}\right)=2^{-n}$. Assume that the partitions $\mathscr{P}_{n}=\left\{A_{n}^{i}, i=1, \ldots, 2^{n}\right\}$ are independent. Let $\mathscr{F}_{n}=\sigma\left(X_{1}, \ldots, X_{n}\right) .\left(X_{n}, \mathscr{F}_{n}\right)$ is an amart, but not a martingale in the limit or a game fairer with time, because if $n<m$, then for each $\omega,\left(E^{\mathscr{G}_{n}} X_{m}\right)(\omega)=$ $E X_{m} \rightarrow 0$, hence for $n$ large,

$$
\sup _{m>n}\left|E^{\mathscr{F}_{n}} X_{m}-X_{n}\right| \geqslant 2^{-1}
$$

To obtain a similar example in an arbitrary Banach space $E$, approximate, by the theorem of Dvoretzky (the weaker form proved in [17] is sufficient), groups of vectors $\left\{e_{n}^{i}, i=1, \ldots, 2^{n}\right\}$ by vectors in $E$; for details see [10].

(d) There are natural variants of conditions (ii) and (iv) in Theorem 2 which cannot be satisfied by any class of sequences of integrable random variables which is larger than the class of amarts. In other terms, it is not possible to generalize martingales beyond amarts, and still retain a certain natural form of the optional sampling theorem, and a very weak form of the Riesz decomposition. To be more precise, let $\underline{A}$ be the class of sequences of random variables $\left(X_{n}\right)$ such that $\left(X_{n}, \sigma\left(X_{1}, \ldots, X_{n}\right)\right)$ is an amart. Let $\underline{C}$ be another class of sequences of random variables such that (a) $\underline{A} \subset \underline{C}$; (b) if $\left(X_{n}\right) \in \underline{C}$, then $\left(X_{\tau_{n}}\right) \in \underline{C}$ for each increasing sequence $\left(\tau_{n}\right)$, such that $\tau_{n} \in T$, $\tau_{n} \geqslant n$; (c) $\left(X_{n}\right) \in \underline{C} \Rightarrow X_{n}=Y_{n}+Z_{n}$ where $Y_{n}$ is a martingale and $E Z_{n} \rightarrow$. Then $\underline{A}=\underline{C}$.

Proof. Let $\left(X_{n}\right) \in \underline{C}, \hat{X}_{n}=X_{\tau_{n}} ;$ then $\left(\hat{X}_{n}\right) \in \underline{C} \Rightarrow \hat{X}_{n}=Y_{n}+Z_{n} \Rightarrow E X_{\tau_{n}}$ $=E \hat{X}_{n}=E Y_{n}+E Z_{n} \rightarrow$.

This implies that $\left(X_{n}\right)$ is an amart, because, looking at subsequences, it is 
easy to see that in the definition of an amart it suffices to assume $E X_{\tau_{n}} \rightarrow$ for all sequences $\tau_{n} \uparrow$ with $\tau_{n} \geqslant n$.

Condition (c) above is, of course, equivalent with the assumption: $E X_{n} \rightarrow$; we preferred to state it in the form of a "weak" Riesz decomposition in order to connect it with Theorem 2(ii).

\section{REFERENCES}

1. D. G. Austin, G. A. Edgar and A. Ionescu Tulcea, Point-wise convergence in terms of expectations, Z. Wahrscheinlichkeitstheorie und Verw. Gebiete 30 (1974), 17-26.

2. A. Bellow, On vector-valued asymptotic martingales, Proc. Nat. Acad. Sci. U.S.A. 73 (1976), 1798-1799.

3. L. H. Blake, A generalization of martingales and two subsequent convergence theorems, Pacific J. Math. 35 (1970), 279-283.

4. Further results concerning games which become fairer with time, J. London Math. Soc. (2) 6(1973), 311-316.

5. D. L. Burkholder, Successive conditional expectations of an integrable function, Ann. Math. Statist. 33 (1962), 887-893.

6. R. V. Chacon, $A$ "stopped proof of convergence", Advances in Math. 14 (1974), 365-368.

7. R. V. Chacon and L. Sucheston, On convergence of vector-valued asymptotic martingales, $\mathrm{Z}$. Wahrscheinlichkeitstheorie und Verw. Gebiete 33 (1975), 55-59.

8. G. A. Edgar and L. Sucheston, Amarts: a class of asymptotic martingales. A. Discrete parameter. B. Continuous parameter, J. Multivariate Analysis 6 (1976), 193-221; 572-591.

9. und verw. Gebiete 36 (1976), 85-92.

10. On vector-valued amarts and dimension of Banach spaces, Z. Wahrscheinlichkeitstheorie und Verw. Gebiete 39 (1977), 213-216.

11. U. Krengel and L. Sucheston, On amarts, semiamarts, and processes with finite values, Advances in Prob. (to appear).

12. B. J. McCabe and L. A. Shepp, On the supremum of $S_{n} / n$, Ann. Math. Statist. 41 (1970), 2166-2168.

13. A. G. Mucci, Limits for martingale-like sequences, Pacific J. Math. 48 (1973), 197-202.

14. __ Another martingale convergence theorem, Pacific J. Math. 64 (1976), 539-541.

15. J. Neveu, Discrete parameter martingales, North-Holland, Amsterdam, 1975.

16. R. Subramanian, On a generalization of martingales due to Blake, Pacific J. Math. 48 (1973), 275-278.

17. L. Tzafriri, On Banach spaces with unconditional bases, Israel J. Math. 17 (1974), 84-93.

Department of Mathematics, Ohio State University, Columbus, Oho 43210 